\title{
IMPACTO DA HERBIVORIA POR CAPRINOS SOBRE AS POPULAÇÕES NATURAIS DE Bromelia laciniosa MART. EX SCHULT. F. (Bromeliaceae) ${ }^{1}$
}

\author{
Fabio da Silva do Espírito Santo², Jefferson Rodrigues Maciel ${ }^{3}$ e José Alves de Siqueira Filho ${ }^{4}$
}

RESUMO - Este estudo teve como objetivo quantificar o impacto da herbivoria por caprinos sobre as populações naturais de Bromelia laciniosa Mart. ex Schult. f. (Bromeliaceae), espécie endêmica da Caatinga. Os estudos de campo foram desenvolvidos na localidade de Caboclo (8²8'56,4"S, 4056'6,9"W, 588 m alt.), Município de Afrânio, Pernambuco. Essa área é considerada de alta importância biológica e, portanto, prioritária para a conservação. A análise quantitativa desse impacto foi realizada a partir do inventário de 10 parcelas com $2.000 \mathrm{~m}^{2}$, ao longo de uma trilha pré-existente na área de estudo, e consistiu em contabilizar os indivíduos de B. laciniosa em floração e o porcentual de indivíduos que tiveram suas inflorescências forrageadas pelos caprinos. Adicionalmente, também foram obtidas informações referentes à fenologia, à morfologia floral e às taxas de formação de frutos e sementes dessa Bromeliaceae. Verificou-se que, dos 246 indivíduos inventariados, $65,8 \%$ tiveram suas inflorescências forrageadas por esses animais. A estimativa do número de flores consumidas foi de 8.748 e o número provável de frutos e sementes que deixaram de ser produzidos, de aproximadamente 7.227 e 252.945, respectivamente. O forrageamento das inflorescências diminuiu as taxas de formação de frutos e sementes, podendo, desse modo, interferir diretamente na conservação dessa bromélia.

Palavras-chave: Caprinocultura extensiva, Forrageamento e Conservação.

\section{IMPACT OF HERBIVORY BY GOATS ON NATURAL POPULATIONS OF Bromelia laciniosa MART. EX SCHULT. F. (Bromeliaceae)}

\begin{abstract}
The objective of this study was to quantify the impact of herbivory by goats on natural populations of Bromelia laciniosa Mart. ex Schult. f. (Bromeliaceae), an endemic species to the Caatinga biome. Field studies were conducted Caboclo (8'28'56,4'S, 4056'6,9"'W, 588 m alt.), municipality of Afrânio, Pernambuco state. This area is regarded as being highly biologically important and therefore it is a priority area for conservation. The quantitative analysis of this impact was based on the inventory of 10 plots of $2.000 \mathrm{~m}^{2}$, along a pre-existing path in the study area, and consisted in doing the accounting of flowering B. laciniosa individuals and the percentage of individuals which had their flowers foraged by goats. Additionally, we also obtained information on the phenology, floral morphology and fruit and seed set rates of this bromeliad. It was found that out of the 246 individuals surveyed, $65.8 \%$ had their flowers foraged by these animals. The estimate of consumed flowers was 8,748 and the likely number of fruit and seeds that thus could not develop were approximately 7,227 fruits and 252,945 seeds. Foraging of inflorescences by goats reduces fruit and seed set rates and it may thus directly interfere with the conservation of this bromeliad.
\end{abstract}

Keywords: Goat husbandry, Foraging and Conservation.

\footnotetext{
${ }^{1}$ Recebido em 20.08.2009 e aceito para publicação em 15.11.2011.

${ }^{2}$ Universidade Estadual de Feira de Santana, UEFS, Brasil. E-mail: <fse.santo@yahoo.com.br>

${ }^{3}$ Jardim Botânico do Recife, JBR, Brasil. E-mail: <Jeff.r.maciel@gmail.com>.

${ }^{4}$ Fundação Universidade Federal do Vale do São Francisco, UNIVASF, Brasil. E-mail: <jose.siqueira@univasf.edu.br>.
} 


\section{INTRODUÇÃO}

O uso não sustentável dos recursos naturais da Caatinga vem ocasionando alterações drásticas em suas paisagens (MAIA et al., 2006; DRUMOND et al., 2008), e cerca de $60 \%$ de sua área já foi alterada (CASTELLETTI et al., 2003). As atividades agropecuárias são a principal causa da fragmentação desse bioma (BARBOSA et al., 2005), sendo a pecuária considerada o principal fator de degradação ambiental, uma vez que é responsável pela diminuição da diversidade biológica da vegetação nativa e da fauna associada (SAMPAIO et al., 1994).

Nas regiões Semiáridas do planeta, a caprinocultura extensiva é considerada importante agente promotor de desertificação (FAO, 1993). Porém, na Caatinga essa opinião só é aceita por alguns pesquisadores, quando as altas taxas de lotação de caprinos são associadas a ações antrópicas, como retirada de lenha e utilização para pastos, agricultura e outras. Essas elevadas taxas de lotação são insuficientes para causar a desertificação do ecossistema, resultando apenas no empobrecimento e redução do porte da vegetação (LEAL et al., 2003).

O Brasil possui cerca de 7.109.052 milhões de caprinos, e o Nordeste, principalmente a região semiárida coberta por vegetação de caatinga, é responsável por 91\% desse rebanho, sendo o Estado de Pernambuco detentor de 14,6\% do plantel nacional (IBGE, 2006). O sistema de criação extensiva, em que os animais permanecem soltos durante todo o ano, é o mais praticado na região, com vários projetos governamentais e instituições financeiras que estimulam a criação de caprinos, por se tratar de atividade de grande importância econômica (LEAL et al., 2003).

Bromelia laciniosa Mart. ex. Schult. f. (Bromeliaceae), conhecida popularmente como macambira, é uma espécie endêmica e de ampla distribuição na Caatinga. Apresenta flores vistosas - que atraem ampla gama de visitantes florais - e frutos dispersos por mamíferos de pequeno a médio porte (sensu ROOSMALEN, 1985). A espécie geralmente forma extensos aglomerados, servindo como sítio para o recrutamento de plântulas de diversas espécies florestais (ROCHA et al., 1997). Portanto, pode ser considerada como uma espécie-chave nas comunidades vegetais da Caatinga. Bessa (1982), reconhecendo o perigo eminente de extinção dessa Bromeliaceae, em razão do seu potencial forrageiro, sugeriu estudos destinados à conservação da espécie como forma de garantir a manutenção das suas populações naturais.
Nesse contexto, este estudo teve por objetivo avaliar o impacto da herbivoria por caprinos sobre as populações de B. laciniosa, ocorrentes em área prioritária para a conservação da Caatinga, de modo a criar subsídios científicos para a elaboração de um plano de manejo adequado que garanta o desenvolvimento das atividades agropecuárias associado à conservação da biodiversidade.

\section{MATERIAL E MÉTODOS}

Os estudos de campo foram conduzidos na localidade de Caboclo (8²8'56,4”S, 4056’6,9”O, 588 m altitude), no Município de Afrânio, Pernambuco (Figura 1). De acordo com o Projeto de Conservação e Utilização Sustentável da Diversidade Biológica Brasileira (PROBIO), através do subprojeto “Avaliações e Ações Prioritárias para a Conservação da Biodiversidade do Bioma Caatinga”, a área é considerada de alta importância biológica e, portanto, prioritária para essa conservação (BRASIL, 2002).

A região apresenta conjunto florístico particular, agrupando várias espécies vegetais endêmicas e de distribuição restrita, como Handroanthus spongiosus (Rizzini) S. Grose, Anemopaegma ataide Gentry - ambas Bignoniaceae - e Ipomoea longistaminea O’Donell (Convolvulaceae). Além da grande riqueza florística, a área possui número considerável de espécie de répteis, anfíbios e aves, sendo recomendadas ações de proteção integral (BRASIL, 2002).

A área de estudo apresentava fitofisionomia de Savana Estépica Arborizada em bom estado de conservação e está integrada ao norte da ecorregião denominada Depressão Sertaneja Meridional. Essa ecorregião possui a paisagem mais típica do Semiárido nordestino e ocupa a maior parte do centro sul da Caatinga (VELLOSO et al., 2002). O clima da localidade de Caboclo é semiárido quente, com temperatura média anual de $25^{\circ} \mathrm{C}$ e precipitação anual variando entre 500 e $800 \mathrm{~mm}$.

Foram alocadas 20 parcelas de 20 x $100 \mathrm{~m}$ ao longo de uma trilha pré-existente na área de estudo, dispostas perpendiculares à trilha, sequencialmente à direita e à esquerda. Após as demarcações, foram sorteadas e inventariadas 10 parcelas, totalizando uma área de dois hectares.

Os estudos referentes à fenologia de $B$. laciniosa foram conduzidos entre novembro de 2005 e março de 2007, sendo registradas informações referentes 

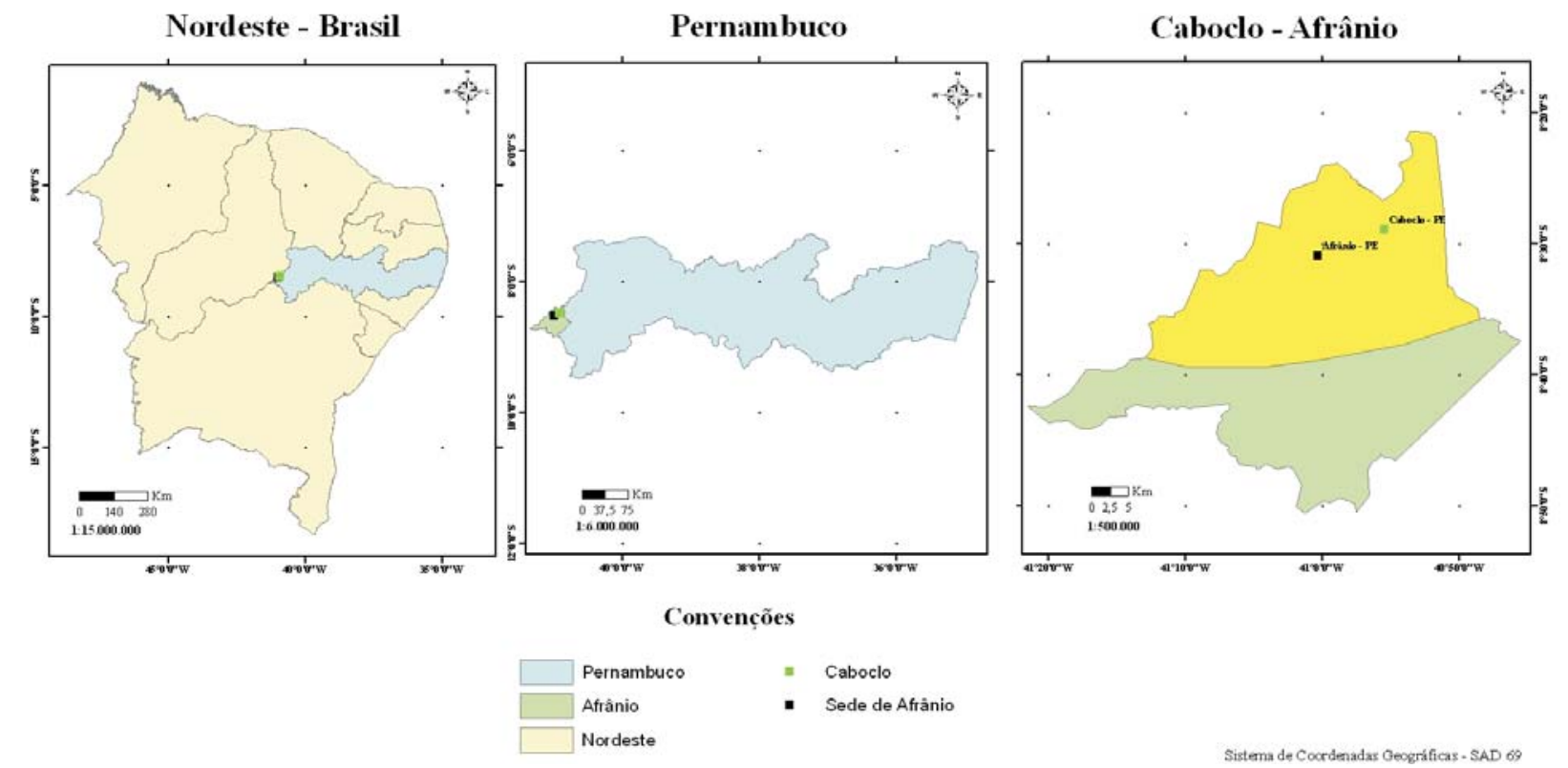

Figura 1 - Localização do povoado de Caboclo, Município de Afrânio, Pernambuco. ( $\square$ ) Área de alta importância biológica da Caatinga.

Figure 1 - Location of Caboclo village, municipality of Afrânio, Pernambuco. ( $\square$ ) Caatinga area of high biological importance.

ao período de floração e frutificação, ao tamanho das inflorescências e ao número de flores/ inflorescência, bem como às taxas de formação de frutos e sementes dessa bromélia. O material botânico de B. laciniosa foi herborizado conforme a metodologia convencional (FIDALGO; BONONI, 1989) e depositado no Herbário do Vale do São Francisco - HVASF (SF 1535), da Universidade Federal do Vale do São Francisco.

A amostragem consistiu em quantificar nas parcelas inventariadas todos os indivíduos de B. laciniosa em floração, sendo registrado o número de plantas que tiveram suas inflorescências forrageadas pelos caprinos. A partir dos experimentos relacionados ao sistema reprodutivo, foi possível estimar o total de flores consumidas por esses animais e a quantidade de frutos e sementes que deixaram de ser produzidos em razão dessa herbivoria.

\section{RESULTADOS}

Bromelia laciniosa é uma espécie terrestre, caracterizada por apresentar porte herbáceo, com folhas dispostas em rosetas, cujo centro adquire coloração rósea durante a floração. Na área de estudo, floresce anualmente entre novembro e janeiro e frutifica entre janeiro e março, sendo encontrada em geral em densas aglomerações.

Essa bromélia apresenta inflorescência ereta, paniculada, rósea, medindo 27,77 cm (DP $= \pm 4,24$, $\mathrm{N}=20$ ), com escapo floral fibroso e carnoso (Figura 2A). O número de flores/inflorescência foi de 54 flores ( $D P= \pm 13, N=30$ ) e a taxa de formação de frutos sob condições naturais, de $82,61 \%$. Os frutos são do tipo baga angulosa, amarelos quando maduros, exalam odor intenso e agridoce e apresentam cerca de 35 sementes $(\mathrm{DP}= \pm 12, \mathrm{~N}=22)$.

Nas parcelas inventariadas foram registrados 246 indivíduos de B. laciniosa em floração, os quais, juntos, produziram, em média, 13.284 flores. Através do inventário realizado, observou-se que $65,8 \%$ dessas plantas tiveram suas inflorescências forrageadas pelos caprinos. Levando-se em consideração o número de flores/ inflorescência e a taxa de formação de frutos de $B$. laciniosa, pode-se inferir que, em média, 8.748 flores foram consumidas por esses animais e que, em razão dessa herbivoria, aproximadamente 7.227 frutos e 252.945

Revista Árvore, Viçosa-MG, v.36, n.1, p.143-149, 2012 

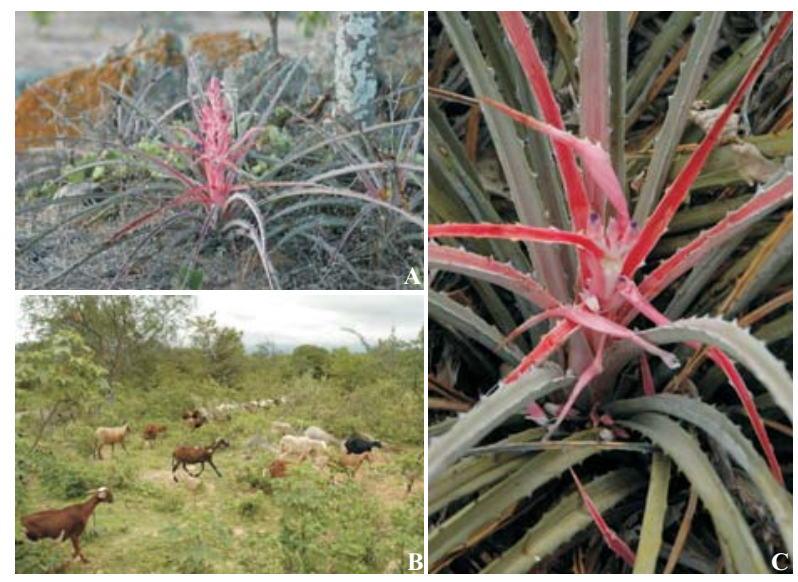

Figura 2 - A: Indivíduo de Bromelia laciniosa Mart. ex Schult. f. (Bromeliaceae) em floração; B: Criação extensiva de caprinos na localidade de Caboclo, Município de Afrânio, Pernambuco; eC: Indivíduo de B. lacniosa após o forrageio dos caprinos.

Figure 2 - A: Flowering individual of Bromelia laciniosa Mart. exSchult.f. (Bromeliaceae); B: Goat husbandry in Caboclo, municipality of Afrânio, Pernambuco; and C: Individual of B. lacniosa after foraging by goats.

sementes deixaram de ser produzidos. Esses animais alimentam-se de toda a inflorescência, deixando visíveis os sinais da herbivoria (Figura 2C).

No local de estudo, os produtores rurais criavam seus animais de forma extensiva, sendo os rebanhos basicamente compostos por animais de corte do tipo “Sem Raça Definida” - SRD (Figura 2B). Os poucos produtores que criavam seus animais de forma semiextensiva destinavam suas atividades para a produção de leite, sendo este beneficiado em cooperativas e comercializado na forma de derivados de leite na região.

Além de B. laciniosa, na localidade de Caboclo diversas outras espécies vegetais eram forrageadas pelos caprinos. Entre elas, merecem destaque Neoglaziovia variegata Mez. (Bromeliaceae) e Pilosocereus gounellei (Weber) Byles \& Rowley (Cactaceae), por serem endêmicas da Caatinga. Em ambas as espécies, esses animais forrageiam flores e frutos, e em $P$. gounellei os caprinos também se alimentam dos cladódios, apesar dos proeminentes espinhos.

\section{DISCUSSÃO}

O porcentual de forrageamento evidencia o elevado grau de comprometimento em que as populações de B. laciniosa se encontravam quando submetidas à ação dos caprinos. Essa herbivoria também afetava, de forma direta, a guilda de polinizadores que utilizam os recursos florais de $B$. laciniosa, como beija-flores, borboletas e abelhas (Obs. pess.). É provável também que existam consequências negativas sobre o fluxo gênico nas populações e no incremento da reprodução assexuada a partir de estolões, uma vez que essa herbivoria diminui a oferta de pólen e, portanto, também a polinização cruzada da espécie.

Tradicionalmente, as folhas e o pseudocaule de B. laciniosa, quando queimados, são utilizados para a alimentação de bovinos, caprinos e suínos, sendo em tempo de seca acentuada também utilizados na fabricação de farinha (LIMA, 1996). Na área de estudo, só foi registrada a utilização das inflorescências in vivo pelos caprinos, não sendo, portanto, utilizadas pelos criadores práticas de queima das folhas para serem consumidas por esses animais, o que também não foi observado em outras áreas cobertas por vegetação de caatinga (LIMA, 1996; PEREIRA et al., 2001).

Durante a estação seca, a serrapilheira, oriunda de árvores e arbustos, apresenta-se como alimento mais importante para os rebanhos da região Semiárida brasileira (KIRMSE; PROVENZA, 1982). Contudo, no final desse período tal recurso se torna escasso (ALBUQUERQUE, 2001), sendo os animais obrigados a desenvolver outras estratégias de alimentação, como o forrageamento de folhas, flores, frutos, sementes, brotos, raízes, tubérculos e cascas de diversas espécies vegetais (MESQUITA et al., 1989; MEDEIROS et al., 2000).

$\mathrm{Na}$ Caatinga, diversas espécies vegetais se encontram seriamente ameaçadas de extinção, em consequência de ações antrópicas, sendo essa situação particularmente dramática quando se trata de espécies endêmicas (BRASIL, 2008). Segundo Araújo-Filho et al. (1996, 1999a, b), até 70\% das espécies herbáceas e lenhosas desse bioma podem ser utilizadas na alimentação de caprinos. Em regiões onde a disponibilidade de água e alimento é limitada, os caprinos desenvolvem diversas habilidades digestivas, como a utilização de fibras vegetais na dieta alimentar e a economia de água e nitrogênio (MEDEIROS et al., 2000). 
De acordo com Leal et al. (2003), em estudo preliminar realizado na região de Xingó, nos Estados da Bahia, de Alagoas e Sergipe, os caprinos alimentam-se de 52 espécies vegetais da Caatinga, sendo utilizadas na sua alimentação tanto as estruturas vegetativas quanto reprodutivas, que apresentam, portanto, notável elasticidade nutricional. Algumas espécies relatadas neste estudo, como Ziziphus joazeiro Mart. (Rhamnaceae), Tacinga palmadora (Britton \& Rose), N. P. Taylor \& Stuppy (Cactaceae), Spondias tuberosa Arruda (Anacardiaceae) e Erythrina velutina Wild. (Fabaceae), são comuns na localidade de Caboclo e também, provavelmente, forrageadas por esses animais.

Segundo Lima e Sidersky (2002), Cavalcanti e Resende (2004) e Silva et al. (2005), em pesquisas também desenvolvidas na Caatinga, nos períodos de seca prolongada, diversas espécies de Cactaceae, como Cereus jamacaru P. DC. e Plosocereus gounelley, também são utilizadas como forrageiras pelos caprinos.

Leal et al. (2003) propuseram que a herbivoria é capaz de alterar padrões de abundância e distribuição geográfica das espécies lenhosas da Caatinga, sendo as que apresentam ciclo reprodutivo parcial ou completo na estação seca mais suscetíveis às mudanças na densidade populacional. A partir dos resultados, é razoável acreditar que outras espécies herbáceas, assim como B. laciniosa, que florescem e, ou, frutificam na estação seca, também corram o risco de redução das suas populações naturais e de consequente extinção local em virtude da pecuária extensiva.

\section{CONCLUSÕES}

O elevado porcentual de inflorescências consumidas e a estimativa da quantidade de frutos e sementes que deixou de ser produzidos em razão da herbivoria por caprinos sugerem o alto impacto dessa atividade sobre as populações naturais de B. laciniosa, ainda não quantificado para outras espécies do bioma Caatinga. Portanto, estudos com outros grupos vegetais são necessários para ampliar o conhecimento sobre o efeito dessa atividade agropecuária na manutenção das espécies vegetais na Caatinga.

\section{AGRADECIMENTOS}

Ao Conselho Nacional de Desenvolvimento Científico e Tecnológico (CNPq), pela bolsa ao primeiro autor; à Fundação O Boticário de Proteção à Natureza
(FBPN/PICN - nº673_2005.2), pelo auxílio financeiro; à Universidade Federal do Vale do São Francisco (UNIVASF), através do LAPEVALE (Laboratórios de Pesquisa do Vale), pela logística nos estudos de campo; ao Ricardo R. D. Ramos, pela confecção do mapa (UNIVASF); e à Gláucia Suêrda G. do Nascimento (UNIVASF), pelo auxílio nas atividades de Campo.

\section{REFERÊNCIAS}

\section{ALBUQUERQUE, S. G. O bioma caatinga representado na cultura popular nordestina. Petrolina: Embrapa Semi-Árido, 2001. 38p. (Embrapa Semi-Árido. Documentos, 166).}

ARAÚJO-FILHO, J. A. et al. Composição botânica e química da dieta de ovinos e caprinos em pastoreio combinado na região dos Inhamus, Ceará. Revista da Sociedade Brasileira de Zootecnia, v.25, n.3, p.383-395, 1996.

ARAÚJO-FILHO, J. A.; BARBOSA, T. M. L.; CARVALHO, F. C. Sistema de produção silvipastoril para o semi-árido nordestino. Boletim da Embrapa, v.29, p.1-2, 1999a.

ARAÚJO-FILHO, J. A. et al. Sistema de produção agrossilvipastoril para o semi-árido nordestino. Sobral: Boletim da Embrapa, v.30, p.1-2, 1999b.

BARBOSA, M. R. V. et al. Estratégias para conservação da biodiversidade e prioridades para a pesquisa científica no bioma Caatinga: Suporte a estratégias regionais de conservação. In: ARAÚJO, F. S.; RODAL, M. J. N.; BARBOSA, M. R. V. (Orgs.). Análise das variações da biodiversidade do bioma Caatinga. Brasília: Ministério do Meio Ambiente, 2005. p.415-429.

BESSA, M. N. A macambira: Bromelia forrageira. 2.ed. Natal: EMPARN, 1982. 135p.

BRASIL. Ministério do Meio Ambiente. Biodiversidade brasileira: avaliação e identificação de áreas prioritárias para conservação, utilização sustentável e repartição de benefícios da biodiversidade brasileira. Brasília: 2002. 404p.

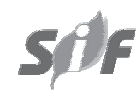

Revista Árvore, Viçosa-MG, v.36, n.1, p.143-149, 2012 
BRASIL. Ministério do Meio Ambiente. Instrução Normativa 06 de 23 de setembro de 2008. Disponível em: <http:// www.mma.gov.br/port/sbf/fauna/flora/pdf/ lista 2008.pdf $>$. Acesso em: 04 dez. de 2008.

CASTELLETTI, C. H. M. et al. Quanto ainda resta da Caatinga? Uma estimativa preliminar. In: LEAL, I. R.; TABARELLI, M.; SILVA, J. M. C. (Eds.). Ecologia e conservação da caatinga. Recife: Universidade Federal de Pernambuco, 2003. p.719-734.

CAVALCANTI, N. B.; RESENDE, G. M. Consumo de xiquexique (Pilosocereus gounellei (A. Weber ex K. Schum.) Bly. ex Rowl) por caprinos no semiárido da Bahia. Revista Caatinga, v. 20, n. 1, p. 22-27, 2007

DRUMOND, M. A. Produção e distribuição de biomassa de espécies arbóreas no Semiário brasileiro. Revista Árvore, v.32, n.4, p.665-669, 2008.

FIDALGO, O.; BONONI, V. L. R. (Coords.). Técnicas de coleta, preservação e herborização de material botânico. São Paulo: Instituto de Botânica, São Paulo, 1989. 62p.

FOOD AND AGRICULTURE ORGANIZATION FAO. El papel de los animals domesticos en el control de la desertificacion. Santiago: PNUD/FAO, Oficina Regional de La Fao para América Latina Y el Caribe, 1993. 115p.

\section{INSTITUTO BRASILEIRO DE GEOGRAFIA E ESTATÍSTICA - IBGE. Censo agropecuário: resultados preliminares. Rio de Janeiro: 2006. 146p.}

KIRMSE, R. D.; PROVENZA, F. D. Herbage response to clearcutting caatinga vegetation in Northeast Brazil. In: SIMPÓSIO BRASILEIRO DO TRÓPICO SEMI-ÁRIDO, 1., 1982, Olinda. Anais... Olinda: Embrapa-CPATSA/UFPE, 1982. p.768-772.

Revista Árvore, Viçosa-MG, v.36, n.1, p.143-149, 2012
LEAL, I. R.; TABARELLI, M.; SILVA, J. M. C. Ecologia e conservação da Caatinga: uma introdução ao desafio. In: LEAL, I. R.; TABARELLI, M.; SILVA, J. M. C. (Eds.). Ecologia e Conservação da Caatinga. Recife: Universidade Federal de Pernambuco, 2003. p.8-16.

LIMA, J. L. S. Plantas forrageiras das caatingas: uso e potencialidades. Petrolina: Embrapa-CPATSA;PNE;RBG-KEW, 1996. 24p.

LIMA, M.; SIDERSKY, P. O papel das plantas nativas nos sistemas agrícolas familiares do Agreste da Paraíba. In: Agricultura familiar e agroecologia no Semiárido: avanços a partir do Agreste da Paraíba. Rio de Janeiro: ASPT, 2002. 355p.

MAIA, S. M. F. Impactos de sistemas agroflorestais e convencional sobre a qualidade do solo no semiárido cearense. Revista Árvore, v.30, n.5, p.837-848, 2006.

MEDEIROS, L. P. et al. Caprinos. Teresina: Embrapa Caprinos, 2000. p.26-34.

MESQUITA, R. C. M.; LEITE, E. R.; ARAÚJOFILHO, J. A. 1989. Estacionalidade da dieta de pequenos ruminantes em ecossistemas de Caatinga. In: CURSO DE MELHORAMENTO E MANEJO DE PASTAGEM NATIVA NO TRÓPICO SEMI-ÁRIDO. Teresina: Embrapa/CPAMN/SPI, 1989. p.59-82.

PEREIRA, I. M. et al. Regeneração natural em um remanescente de Caatinga sob diferentes níveis de perturbação, no Agreste paraibano. Acta Botanica Brasilica, v.15, n.3, p.413-426, 2001.

ROCHA, C. F. D. et al. Bromélias: ampliadoras da biodiversidade. Bromélia, v.4, n.4, p.7-10, 1997.

van RoOSMALEN, M. G. M. Fruits of the Guianan Flora. Wageningen: University of Utrecht Press/Veenman, 1985. 517p.

SAMPAIO, E. V. S. B. et al. Caatingas e Cerrados do NE - biodiversidade e ação antrópica. In: CONFERÊNCIA NACIONAL E SEMINÁRIO LATINO-AMERICANO DA DESERTIFICAÇÃO, 1994, Fortaleza. Anais... Fortaleza: 1994. p.1-15. 
SILVA, J. G. M. et al. Xiquexique

(Pilosocereus gounellei (A. Weber ex K. Schum.) Bly. Ex Rowl.) em substituição à silagem de sorgo (Sorghum bicolor L.

Moench) na alimentação de vacas leiteiras.

Revista Brasileira de Zootecnia, v.34, n.4, p.1408-1417, 2005.
VELLOSO, A. L.; SAMPAIO, E. V. S. B.; PAREYN, F. G. C. (Eds). Ecorregiões propostas para o Bioma Caatinga. Resultados do Seminário de Planejamento Ecorregional da Caatinga. Aldeia-PE. 28 a 30 de novembro de 2001. Recife: Associação Plantas do Nordeste / The Nature Conservancy do Brasil, 2002. 76p. 